Mathematical Modelling and Analysis

VOLUME 4, 1999, PAGES 163-173

(C) 1999 Technika

\title{
THE ROOT CONDITION FOR THE THIRD ORDER POLYNOMIALS
}

\section{A. ŠTIKONAS}

Vilnius Gediminas Technical University, Vytautas Magnus University, Institute of Mathematics and Informatics

Akademijos 4, 2600, Vilnius, Lithuania

E-mail: stikonas@fm.vtu.lt

Received October 1, 1999

\begin{abstract}
This paper deals with a root condition for polynomial of the second and third order. We prove the root criterion for such polynomial with complex coefficients and find regions for the root condition in the special coefficients' phase space.
\end{abstract}

A stability concept for discrete problems is of the most importance in the numerical analysis. Since the stability and consistency imply convergence. The von Neumann stability definition is used for problems with constant coefficients. It requires that all eigenvalues of the characteristic equation (or the amplification matrix) be in the closed unit disc and the ones on the unit circle be simple [11]. For finite-difference schemes we can get necessary stability conditions from a spectral (von Neumann) stability analysis [1]. In particular, von Neumann's condition is necessary for stability in $L_{2}$. Often these necessary conditions are sufficient conditions for linear finite-difference schemes too. The definition of spectral stability appears when we investigate stability of numerical integration methods (Runge-Kutta, multistep methods) for ordinary differential equations $[1 ; 4 ; 6 ; 5 ; 13]$ and partial differential equations $[1$; $12]$.

\section{ROOT CONDITION}

Consider a complex polynomial

$$
f(z)=P_{n}(z)=a_{n} z^{n}+a_{n-1} z^{n-1}+\cdots+a_{1} z+a_{0},
$$


with coefficients $a_{i} \in \mathbb{C}$, where $\mathbb{C}$ - is a set of complex numbers. If $a_{n} \neq 0$ then such polynomial has $n$ roots $q_{i} \in \mathbb{C}, i=1, \ldots, n$, exactly.

Now we formulate the root condition for polynomial (1.1) (see Fig. 1) [1; $13]$ :

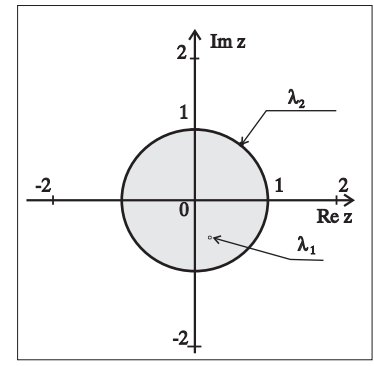

Figure 1. Root condition.

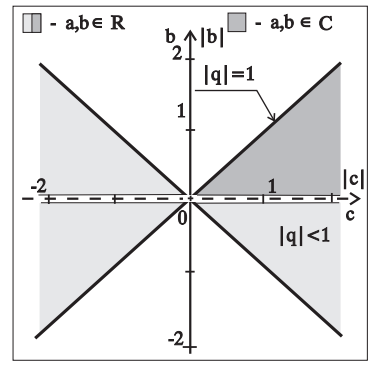

Figure 2. Criterion for linear polynomial $(b \neq 0)$.

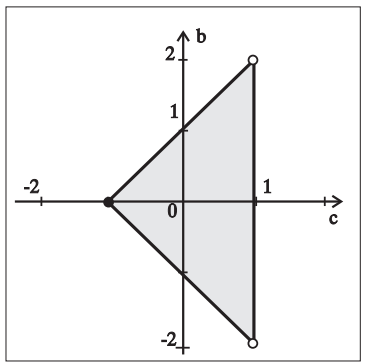

$\beta=0$

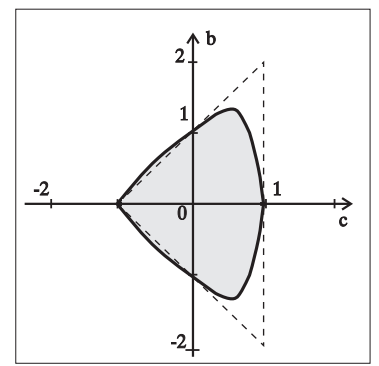

$0<\beta<\frac{\pi}{2}, \frac{\pi}{2}<\beta<\pi$

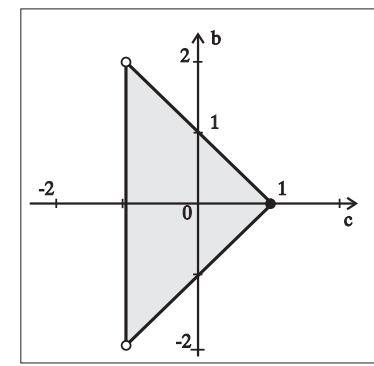

$\beta=\frac{\pi}{2}$

Figure 3. Criterion for $C=c$ and $B=b e^{\beta \mathbf{i}}$ with $b, c \in \mathbb{R}, 0 \leqslant \beta<\pi$.

Definition 1.1. Polynomial $P_{n}$ satisfies the root condition if all roots of this polynomial are in the closed unit disc of complex plane and those roots of magnitude 1 are simple.

Usually we use two-level or three-level finite-difference schemes for nonstationary partial differential equations. In this case we get linear polynomial $P_{1}(z)=b z+c$ or the second order polynomial $P_{2}(z)=a z^{2}+b z+c$. There are no problems with the linear equation because it has only one root $q$ (if $b \neq 0$ ). We must check the magnitude of this root. If it is less or equal than 1 then polynomial satisfies the root condition. Thus, in this case we have equality

$$
A \equiv\left\{(c, b) \in \mathbb{C}^{2},|q| \leqslant 1\right\}=\{|c| \leqslant|b|, b \neq 0\} .
$$

and an obvious criterion for the linear polynomial. Therefore, to check the 
root condition for this polynomial, we simply check whether both coefficients $b$ and $c$ belong to the root condition set $A$ (see Fig. 2 with axes $|c|$ and $|b|$ in the general case and axes $c$ and $b$ in the real polynomial case). If $b=0$ and $c \neq 0$ then there are no roots. If $b=0$ and $c=0$ then all $q \in \mathbf{C}$ are roots of linear equation, i.e. linear polynomial does not satisfy the root condition.

In general case we have no such a simple criterion for polynomial (1.1). Let us denote by $p(p \leqslant n)$ the number of zeros of the polynomial $f(z)$ which are in the unit circle $|z|=1$. One of the ways to determine $p$ is to map the interior $|z|<1$ of the unit circle into the left half of the complex plane $\operatorname{Re} z<0$. Then the number of zeros may be found by using Hurwitz's criterion for a new polynomial $F(z)=z^{n}+A_{1} z^{n-1}+\ldots+A_{n}$ in this domain $[7 ; 8]$.

If we consider only the polynomials of the second order $z^{2}+B z+C=0$, $A=1, B=b \in \mathbb{R}, C=c \in \mathbb{R})$ then the well-known Hurwitz's criterion holds: all (two) roots of real polynomial of the second order are in the unit disk if it's coefficients satisfy the two simple inequalities

$$
|c| \leqslant 1, \quad|b| \leqslant c+1
$$

The set of the points $(c, b)$ satisfying Hurwitz's criterion make a triangle (see Fig. $3, \beta=0$ ). We notice that the double root is on the unit circle, when $c=1$ and $D=b^{2}-4 c=0$, i.e. $|b|=2$.

\section{MAIN RESULTS FOR ROOTS IN THE UNIT CIRCLE}

Let us associate with $f(z)=a_{n} z^{n}+\ldots+a_{1} z+a_{0}$ the conjugate polynomial (by circle $S=\{z \in \mathbb{C}:|z|=1\}$ )

$$
f^{*}(z)=z^{n} \bar{f}(1 / z)=\bar{a}_{0} z^{n}+\bar{a}_{1} z^{n-1}+\cdots+\bar{a}_{n}=\bar{a}_{0} \prod_{j=1}^{n}\left(z-z_{j}^{*}\right),
$$

whose zeros $z_{k}^{*}=1 / \bar{z}_{k}$ are, relative to circle $|z|=1$, the inverses of the zeros $z_{k}$ of $f(z)$.

Now we shall follow Morris Marden's "Geometry of Polynomials" [10]. For $f(z)$ and $f^{*}(z)$ we construct the sequence of polynomials $f_{j}(z)=\sum_{k=0}^{n-j} a_{k}^{(j)} z^{k}$, where $f_{0}(z)=f(z)$ and

$$
f_{j+1}(z)=\bar{a}_{0}^{(j)} f_{j}(z)-a_{n-j}^{(j)} f_{j}^{*}(z), \quad j=0,1, \ldots, n-1 .
$$

Thus,

$$
a_{k}^{(j+1)}=\bar{a}_{0}^{(j)} a_{k}^{(j)}-a_{n-j}^{(j)} \bar{a}_{n-j-k}^{(j)} .
$$

The constant term $a_{0}^{(j)}$ in each polynomial $f_{j}(z)$ is a real number which we denote by $\delta_{j}$ :

$$
\delta_{j+1}=\left|a_{0}^{(j)}\right|^{2}-\left|a_{n-j}^{(j)}\right|^{2}=a_{0}^{(j+1)}, \quad j=0,1,2, \ldots, n-1 .
$$


As to the zeros of these polynomials, Cohn [2] has proved lemma which we present in the compact form due to Marden [9] (in the case when $f(z)$ has no zeros on the circle $S$ ). We formulate this lemma in general case.

Lemma 2.1. If $f_{j}$ has $p_{j}$ zeros interior to the unit circle $S:|z|=1, s_{j}$ zeros on the $S$ and if $\delta_{j+1} \neq 0$, then $f_{j+1}$ has

$$
p_{j+1}=(1 / 2)\left(n-j-s_{j}-\left(n-j-s_{j}-2 p_{j}\right) \operatorname{sign} \delta_{j+1}\right)
$$

zeros interior to $S$. Furthermore, $f_{j+1}$ has the same zeros on $S$ as $f_{j}$.

Remark 2.1. If $\delta_{j+1}>0$ then $f_{j+1}$ has in circle $S$ the same $p_{j}$ of zeros as $f_{j}$, if $\delta_{j+1}<0$ then $f_{j+1}$ has in $S$ the same $n-j-s_{j}-p_{j}$ of zeros as $f_{j}^{*}$,

Schur $[14 ; 15]$ has proved lemma about polynomials in the case $\left|a_{0}\right|<\left|a_{n}\right|$.

Lemma 2.2. If $\left|a_{0}\right|<\left|a_{n}\right|$ then polynomial $f(z)$ has its all zeros in the unit circle $S$ if and only if $f_{1}^{*}(z)$ has its zeros in the unit circle.

Let construct sequence: $P_{k}=\delta_{1} \delta_{2} \ldots \delta_{k}, k=1,2, \ldots, n$. Marden[10] has proved theorem:

Theorem 2.1. For a given polynomial $f(z)=a_{0}+a_{1} z+\cdots+a_{n} z^{n}$, let the sequence $f_{j+1}(z)$ be constructed. Then, if for some $k<n, P_{k} \neq 0$ in $P_{k}=\delta_{1} \delta_{2} \ldots \delta_{k}, k=1,2, \ldots, n$, but $f_{k+1}(z) \equiv 0$, then $f$ has $n-k$ zeros on or symmetric in the circle $S:|z|=1$ at the zeros of $f_{k}(z)$. If $p$ of the $P_{j}, j=1,2, \ldots, k$, are negative, then $f$ has $p$ additional zeros inside $S$ and $q=k-p$ additional zeros outside $S$.

Definition 2.1. Polynomial $g(z)=b_{0}+b_{1} z+\cdots+b_{n} z^{n}$ is self-inversive, if zeros of $g$ on $S$ or symmetric in $S$.

If $f$ (and $f^{*}$ ) is self-inversive then [10] $f=u f,|u|=1$ and coefficients of $f$ satisfy the relations:

$$
b_{n}=u \bar{b}_{0}, b_{n-1}=u \bar{b}_{1}, \ldots b_{0}=u \bar{b}_{n},|u|=1 .
$$

In this case $\left|b_{0}\right|=\left|b_{n}\right|$, and if $\left|b_{0}\right| \neq\left|b_{n}\right|$ then polynomial is not self-inversive. For such polynomial Marden [10] has proved:

Lemma 2.3. If $g$ is a self-inversive polynomial, its derivative $g^{\prime}$ has no zeros on the circle $S:|z|=1$ except at the multiple zeros of $g$. 
Theorem 2.2. If $g(z)$ is a self-inversive polynomial then $g$ has as many zeros in the circle $S$ as the polynomial

$$
g_{1}(z)=\left[g^{\prime}(z)\right]^{*}=\sum_{j=0}^{m-1}(m-j) \bar{b}_{m-j} z^{j}
$$

has. That is, $g$ and $g^{\prime}$ have the same number of zeros for $|z|>1$.

\section{THE ROOT CONDITION FOR COMPLEX POLYNOMIAL OF THE SECOND ORDER}

Let the roots of the second order equation

$$
f(z)=A z^{2}+B z+C=0
$$

be $q_{1}$ and $q_{2}$. We denote the set of coefficients of this polynomial $\widetilde{\mathbb{C}}^{3}=$ $\left\{(A, B, C) \in \mathbb{C}^{3}, A \neq 0\right\}$ and separate some subsets of this set in the following way:

$$
\begin{aligned}
A_{0}=\left\{(A, B, C) \in \widetilde{\mathbb{C}}^{3},\right. & \left.\left|q_{1}\right|<1,\left|q_{2}\right|<1\right\}, \\
A_{1}=\left\{(A, B, C) \in \widetilde{\mathbb{C}}^{3},\right. & \left.\left|q_{1}\right|<1,\left|q_{2}\right|=1\right\}, \\
A_{11}=\left\{(A, B, C) \in \widetilde{\mathbb{C}}^{3},\right. & \left.\left|q_{1}\right|=\left|q_{2}\right|=1, q_{1} \neq q_{2}\right\}, \\
A_{2}=\left\{(A, B, C) \in \widetilde{\mathbb{C}}^{3},\right. & \left.\left|q_{1}\right|=\left|q_{2}\right|=1, q_{1}=q_{2}\right\} .
\end{aligned}
$$

The root condition holds if the coefficients of the polynomial belong to one of the sets: $A_{0}, A_{1}$ and $A_{11}$. Then a set $A_{R}=A_{0} \cup A_{1} \cup A_{11}$ is the root condition set. The analysis of these sets using theorems for zeros in the unit circle implies[16] lemma for second order polynomials.

Lemma 3.1. The following equalities are valid:

$$
\begin{aligned}
A_{0} & =\left\{|C|^{2}+|\bar{B} A-\bar{C} B|<|A|^{2}\right\}, \\
A_{1} & =\left\{|C|^{2}+|\bar{B} A-\bar{C} B|=|A|^{2},|C|<|A|\right\}, \\
A_{11} & =\{|C|=|A|, \bar{B} A=\bar{C} B,|B|<2|A|\}, \\
A_{2} & =\{|C|=|A|, \bar{B} A=\bar{C} B,|B|=2|A|\} .
\end{aligned}
$$

This result about root condition we formulate as the following theorem.

Theorem 3.1 [The root condition]. The roots of the second order polynomial are in the closed unit disc of complex plane and those roots of magnitude 1 are simple if

$$
A_{R}=\left\{|C|^{2}+|\bar{B} A-\bar{C} B| \leqslant|A|^{2},|B|<2|A|\right\} .
$$


If $a=A$ then the root condition reduces to

$$
A_{R}=\left\{|C|^{2}+|\bar{B}-\bar{C} B| \leqslant 1, \quad|B|<2\right\} .
$$

If $A=1, B=b=\bar{b} \in \mathbb{R}, C=c=\bar{c} \in \mathbb{R}$ then equality (3.3) corresponds to root condition (1.3) in Hurwitz's criterion.

Now we consider (3.1) when $A=1$ in the form

$$
w^{2}+B w+C=0
$$

with $B=b e^{\beta \mathbf{i}}, C=c e^{\beta \mathbf{i}}, \beta, \gamma \in[0,2 \pi), b, c \in \mathbb{R}$. Such form of the complex number $Z=z e^{\varphi \mathbf{i}}, z \in \mathbb{R}, \varphi \in[0, \pi)$ with negative $z$ is equivalent to standard exponential form of complex number $Z=|Z| e^{(\varphi+\pi) \mathbf{i}}$. Thus, every complex number $Z=|Z| e^{\varphi \mathbf{i}}, \varphi \in[0,2 \pi)$ we may write in such form. The root condition set $A_{R}$ for various $\beta$ and $\gamma$ are sophisticated (the case $\gamma=0$ see Fig. 3).

\section{THE ROOT CONDITION FOR COMPLEX POLYNOMIAL OF THE THIRD ORDER}

Let the zeros of the third order polynomial

$$
f(z)=c+b z+a z^{2}+d z^{3}, a, b, c, d \in \mathbb{C}, d \neq 0 .
$$

be $q_{1}, q_{2}$ and $q_{3}$. We denote the set of coefficients $k=(a, b, c, d)$ of this polynomial $\widetilde{\mathbb{C}}^{4}=\left\{k \in \mathbb{C}^{4}, d \neq 0\right\}$ and separate some $\widetilde{\mathbb{C}}^{4}$ subsets:

$$
\begin{aligned}
A_{0} & =\left\{\left|q_{1}\right|<1,\left|q_{2}\right|<1,\left|q_{3}\right|<1\right\}, \\
A_{D} & =\left\{\left|q_{1}\right| \leqslant 1,\left|q_{2}\right| \leqslant 1,\left|q_{3}\right| \leqslant 1\right\}, \\
A_{1} & =\left\{\left|q_{1}\right|<1,\left|q_{2}\right|<1,\left|q_{3}\right|=1\right\}, \\
A_{11} & =\left\{\left|q_{1}\right|<1, q_{2} \neq q_{3},\left|q_{2}\right|=\left|q_{3}\right|=1\right\}, \\
A_{2} & =\left\{\left|q_{1}\right|<1, q_{2}=q_{3}=q,|q|=1\right\}, \\
A_{111} & =\left\{q_{1} \neq q_{2}, q_{2} \neq q_{3}, q_{1} \neq q_{3},\left|q_{1}\right|=\left|q_{2}\right|=\left|q_{3}\right|=1\right\}, \\
A_{21} & =\left\{q_{1} \neq q_{2}=q_{3}=q,\left|q_{1}\right|=|q|=1\right\}, \\
A_{3} & =\left\{q_{1}=q_{2}=q_{3}=q,|q|=1\right\} .
\end{aligned}
$$

Let be denote

$$
\begin{aligned}
f^{*}= & \bar{d}+\bar{a} z+\bar{b} z^{2}+\bar{c} z^{3}, \\
& A=|c|^{2}-|d|^{2} \in \mathbb{R}, \quad B=c \bar{b}-a \bar{d}, C=c \bar{a}-b \bar{d}, \\
f_{1}= & A+\bar{B} z+\bar{C} z^{2}, f_{1}^{*}=C+B z+A z^{2}, \\
f_{2}= & |A|^{2}-|C|^{2}+(A \bar{B}-B \bar{C}) z, f_{2}^{*}=A B-\bar{B} C+\left(|A|^{2}-|C|^{2}\right) z, \\
f_{3}= & \left(|A|^{2}-|C|^{2}\right)^{2}-|A \bar{B}-B \bar{C}|^{2} .
\end{aligned}
$$


Polynomial is self-inversive if $A=0, B=0, C=0$. So the set $A_{D}=A_{0} \cup$ $A_{1} \cup A_{11} \cup A_{111} \cup A_{2} \cup A_{21} \cup A_{3}$ is the condition that all zeros are in the unit disc.

\begin{tabular}{|l|l|l|l|}
\hline$A_{i}$ & $A_{111}$ & $A_{21}$ & $A_{3}$ \\
\hline$f$ & $\ddots$ & $\ddots$ & \\
\hline$f^{\prime}$ & $\cdots$ & & \\
\hline
\end{tabular}

Figure 4. Self-inversive case.

\begin{tabular}{|l|l|l|l|}
\hline$A_{i}$ & $A_{1}$ & $A_{11}$ & $A_{2}$ \\
\hline$f$ & $\bullet$ & $\bullet$ & \\
\hline$f_{1}^{*}$ & $\bullet$ & \\
\hline
\end{tabular}

Figure 5. Non self-inversive case.

Lemma 4.1. Consider (4.1) polynomial and subsets of $\widetilde{\mathbb{C}}^{4}$ :

$$
\begin{aligned}
\tilde{A}_{0}= & \left\{A<0,|C|^{2}+|A \bar{B}-B \bar{C}|<|A|^{2}\right\}, \\
\tilde{A}_{D}= & \left\{A<0,|C|^{2}+|A \bar{B}-B \bar{C}| \leqslant|A|^{2}\right\} \\
& \cup\left\{A=0, B=0,|b|^{2}+2|b \bar{a}-3 a \bar{d}| \leqslant 9|d|^{2}\right\}, \\
\tilde{A}_{1}= & \left\{A<0,|C|<|A|,|C|^{2}+|A \bar{B}-B \bar{C}|=|A|^{2}\right\}, \\
\tilde{A}_{11}= & \{A<0,|C|=|A|, A \bar{B}=B \bar{C},|B|<2|A|\}, \\
\tilde{A}_{2}= & \{A<0,|C|=|A|, A \bar{B}=B \bar{C},|B|=2|A|\}, \\
\tilde{A}_{111}= & \left\{A=0, B=0,|b|^{2}+2|b \bar{a}-3 a \bar{d}|<9|d|^{2}\right\},
\end{aligned}
$$




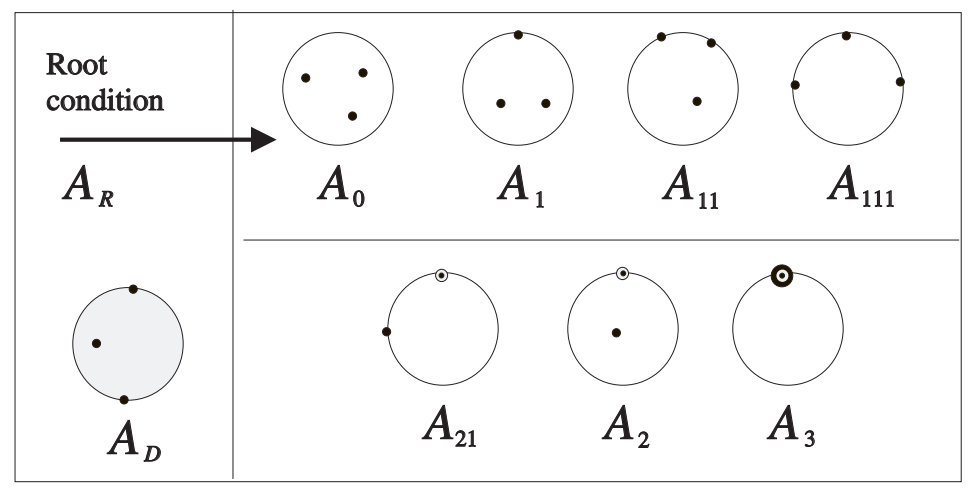

Figure 6. Root condition.

$$
\begin{aligned}
\tilde{A}_{21} & =\left\{A=0, B=0,|b|<3|d|,|b|^{2}+2|b \bar{a}-3 a \bar{d}|=9|d|^{2}\right\} \\
\tilde{A}_{3} & =\{A=0, B=0, b \bar{a}=3 a \bar{d}\} .
\end{aligned}
$$

Then $A_{i}=\tilde{A}_{i}$.

Proof. We prove those equalities in the case $d=1$, because the general case $(d \neq 0)$ we get from lemma if $a, b, c$ replace with $a / d, b / d, c / d$.

From Viete theorem $|c|=\left|q_{1}\right| \cdot\left|q_{2}\right| \cdot\left|q_{3}\right| \leqslant 1$ and $|c|=1$ when $\left|q_{1}\right|=\left|q_{2}\right|=$ $\left|q_{3}\right|=1$.

If $k \in A_{0}$ then $|c|<1$. If $k \in \tilde{A}_{0}$ then $|A|>0$, i.e. $|c|<1$ too. So, from lemma $2.2, f_{1}^{*}$ definition and lema 3.1 (the case $A_{0}$ ) follows equality $A_{0}=\tilde{A}_{0}$.

Now consider polynomial

$$
F_{\varepsilon}(z)=f((1+\varepsilon) z)=(1+\varepsilon)^{3} z^{3}+(1+\varepsilon)^{2} a z^{2}+(1+\varepsilon) b z+c, \varepsilon>0,
$$

which all zeros are in the circle $S$ if and only if polynomial $f$ zeros are in the circle $S_{\varepsilon+1}=\{z:|z|<1+\varepsilon\}$. For such polynomial:

$$
\begin{aligned}
& A_{\varepsilon}=\left|c_{\varepsilon}\right|^{2}-\left|d_{\varepsilon}\right|^{2}=|c|^{2}-(1+\varepsilon)^{6} \rightarrow A, \varepsilon \rightarrow 0, \\
& B_{\varepsilon}=c_{\varepsilon} \bar{b}_{\varepsilon}-a_{\varepsilon} \bar{d}_{\varepsilon}=(1+\varepsilon) c \bar{b}-(1+\varepsilon)^{5} a \rightarrow B, \varepsilon \rightarrow 0, \\
& C_{\varepsilon}=c_{\varepsilon} \bar{a}_{\varepsilon}-b_{\varepsilon} \bar{d}_{\varepsilon}=(1+\varepsilon)^{2} c \bar{a}-(1+\varepsilon)^{4} b \rightarrow C, \varepsilon \rightarrow 0 .
\end{aligned}
$$

If $|c|<1$ then $|A|>0$ and from condition

$$
\left|C_{\varepsilon}\right|^{2}+\left|A_{\varepsilon} \bar{B}_{\varepsilon}-B_{\varepsilon} \bar{C}_{\varepsilon}\right|<\left|A_{\varepsilon}\right|^{2}
$$

we get

$$
|C|^{2}+|A \bar{B}-B \bar{C}| \leqslant|A|^{2}
$$


Coefficients of the polynomials and its zeros are continuously depending from each other. So, we find the set $A_{D} \cap\{|c|<1\}$.

For $|c|=1$ we have

$$
\begin{aligned}
& A_{\varepsilon}=1-(1+\varepsilon)^{6}=-6 \varepsilon(1+o(1)) \rightarrow 0, \varepsilon \rightarrow 0 \\
& C_{\varepsilon}=(1+\varepsilon)^{2}(c \bar{a}-b-b \varepsilon(2+\varepsilon)) \rightarrow c \bar{a}-b, \varepsilon \rightarrow 0
\end{aligned}
$$

If $c \bar{a} \neq b$ then for small $\varepsilon>0$

$$
\left\{\left|C_{\varepsilon}\right|^{2}+\left|A_{\varepsilon} \bar{B}_{\varepsilon}-B_{\varepsilon} \bar{C}_{\varepsilon}\right|<\left|A_{\varepsilon}\right|^{2}\right\}=\varnothing .
$$

In the case $|c|=1, b=c \bar{a}$ we have

$$
\begin{aligned}
& B_{\varepsilon}=a(1+\varepsilon)\left(1-(1+\varepsilon)^{4}\right)=-4 a \varepsilon(1+o(1)), \varepsilon \rightarrow 0, \\
& C_{\varepsilon}=-2 \varepsilon b(1+o(1)), \varepsilon \rightarrow 0,
\end{aligned}
$$

and $A=0, B=0, C=0$, i.d. we have self-inversive polynomial. From (4.2) inequality follows

$$
4 \varepsilon^{2}|a|^{2}(1+o(1))^{2}+8 \varepsilon^{2}(1+o(1))^{2}|3 a-b \bar{a}|<36 \varepsilon^{2}(1+o(1))^{2}, \varepsilon \rightarrow 0,
$$

or

$$
|a|^{2}(1+o(1))^{2}+2(1+o(1))^{2}|3 a-b \bar{a}|<9(1+o(1))^{2}, \varepsilon \rightarrow 0 .
$$

Finally, we get $(\varepsilon \rightarrow 0)$ :

$$
\left\{|a|^{2}+2|3 a-b \bar{a}| \leqslant 9\right\}=A_{D} \cap\{|c|=1\},
$$

i.e. $A_{D}=\tilde{A}_{D}=\tilde{A}_{0} \cup \tilde{A}_{1} \cup \tilde{A}_{2} \cup \tilde{A}_{3} \cup \tilde{A}_{11} \cup \tilde{A}_{21} \cup \tilde{A}_{111}$.

If $A=0, B=0$ then $C=0$. So, $\tilde{A}_{D} \backslash \tilde{A}_{0}=A_{D} \backslash A_{0}=A_{S} \cup A_{N S}$, where

$$
\begin{aligned}
A_{S} & =\left\{A=0, B=0,|b|^{2}+2|b \bar{a}-3 a \bar{d}| \leqslant 9|d|^{2}\right\} \\
A_{N S} & =\left\{A<0,|C|^{2}+|A \bar{B}-B \bar{C}|=|A|^{2}\right\}
\end{aligned}
$$

and $A_{S}=A_{111} \cup A_{21} \cup A_{3}, A_{N S}=A_{1} \cup A_{11} \cup A_{2}$.

Let consider self-inversive case. From lemma 2.3 and theorem $2.2 f^{\prime}$ has all zeros in circle $S$ (see Fig. 4). So, we investigate polynomial $f^{\prime}=3 z^{2}+2 a z+b$ and from lemma 3.1 get $\tilde{A}_{111} \subset A_{111}, \tilde{A}_{21} \subset A_{21}, \tilde{A}_{3} \subset A_{3}$.

Let consider non self-inversive case. If $0<|c|<1$ From lemma $2.1 f_{1}^{*}$ has all zeros in circle $S$ (see Fig. 5). So, we investigate polynomial $f_{1}^{*}=A z^{2}+B z+C$ and from lemma 3.1 get $\tilde{A}_{1} \cap\{0<|c|<1\} \subset A_{1}, \tilde{A}_{11} \cap\{0<|c|<1\} \subset A_{11}$, $\tilde{A}_{2} \cap\{0<|c|<1\} \subset A_{2}$. 
If $c=0$ then $f(z)=z\left(z^{2}+a z+b\right)$ and one zero is $q_{1}=0$. We investigate zeros of polynomial $F=z^{2}+a z+b$ and get

$$
\begin{aligned}
\tilde{A}_{1} \cap\{c=0\} & =\left\{c=0,|b|<1,|b|^{2}+|\bar{a}-\bar{b} a|=1\right\} \subset A_{1}, \\
\tilde{A}_{21} \cap\{c=0\} & =\{c=0,|b|=1, \bar{a}=\bar{b} a|,| a \mid<2\} \subset A_{21}, \\
\tilde{A}_{2} \cap\{c=0\} & =\{c=0,|b|=1, \bar{a}=\bar{b} a|,| a \mid=2\} \subset A_{2} .
\end{aligned}
$$

So, $\tilde{A}_{1} \subset A_{1}, \tilde{A}_{11} \subset A_{11}, \tilde{A}_{2} \subset A_{2}$.

Finally, we prove $\tilde{A}_{i} \subset A_{i}$, i.e. $\tilde{A}_{i}=A_{i}$.

The root condition set $A_{R}=A_{D} \backslash\left(A_{3} \cup A_{2} \cup A_{21}\right)$ (see Fig. 6).

Theorem 4.1 [The root condition]. Polynomial $f$ of the third order is satisfying root condition if

$$
\begin{array}{r}
A_{R}=\left(\left\{A<0,|C|^{2}+|A \bar{B}-B \bar{C}| \leqslant|A|^{2}\right\}\right. \\
\backslash\{A<0,|C|=|A|, A \bar{B}=B \bar{C},|B|=2|A|\}) \\
\cup\left\{A=0, B=0,|b|^{2}+2|b \bar{a}-3 a \bar{d}|<9|d|^{2}\right\} .
\end{array}
$$

\section{REFERENCES}

[1] N.S. Bachvalov, N.P. Zhidkov and G.M. Kobelkov. Numerical Methods. Nauka, Moscow, 1987. (in Russian)

[2] A. Cohn. Über die Anzahl der Wurzeln einer algebraischen Gleichung in einem reise. Math. Z., 14, 1922, 110-148.

[3] F.R. Gantmaxer. Theory of Matrix. Nauka, Moscow, 1988. (in Russian)

[4] E. Hairer, S.P. Nørsett and G. Wanner. Solving Ordinary Differential Equations I. Nonstiff Problems. Springer Series in Computional Mathematics 8. Springer-Verlag Berlin Heidelberg, 1987.

[5] E. Hairer and G. Wanner. Solving Ordinary Differential Equations II. Stiff and Differential-Algebraic Problems. Springer Series in Computional Mathematics 14. Springer-Verlag Berlin Heidelberg, 1991.

[6] P. Henrici. Applied and computational complex analysis V.1. John Wiley \& Sons, New York, 1974.

[7] A. Hurwitz. Ueber die Bedingungen, unter welchen eine Gleichung nur Wurzeln mit negativen reellen Theilen besitzt. Math. Ann., 46, 1895, 273-284.

[8] A. Hurwitz. Ueber die Bedingungen, unter welchen eine Gleichung nur Wurzeln mit negativen reellen Theilen besitzt. Math. Werke, 2, 533-545.

[9] M. Marden. The number of zeros of a polynomial in a circle. In: Proc. Nat. Acad. Sci. U.S.A., 34, 1948, 15-17.

[10] M. Marden. Geometry of polynomials. American Mathematical Society, Providence, Rhode Island, 2nd edition, 1966.

[11] R.D. Richtmayer and K.W. Morton. Difference methods for initial value problems. Interscience Publishers, New York, 1967.

[12] A.A. Samarskii. Theory of Difference Schemes. Nauka, Moscow, 1989. (in Russian) 
[13] A.A. Samarskii and A.V. Goolin. Numerical Methods. Nauka, Moscow, 1989. (in Russian)

[14] I. Schur. Über Potenzreihen, die in Innern des Einheitskreises beschränkt sind. J. Reine Angew. Math., 147, 1917, 205-232.

[15] I. Schur. Über Polynome, die nur in Innern des Einheitskreis verschwinden, ibid. $J$. Reine Angew. Math., 148, 1918, 122-145.

[16] A. Stikonas. The root condition for polynomial of the second order and a spectral stability of finite-difference schemes for Kuramoto-Tsuzuki equations. Mathematical Modelling and Analysis, 3, 1998, $214-226$.

\section{ŠAKNU SĄLYGA TREČIOS EILĖS DAUGIANARIUI}

\section{A. S̆TIKONAS}

Straipsnyje nagrinėjama šaknų sąlyga kvadratiniam ir kubiniam daugianariui. İrodytas šaknų sąlygos kriterijus daugianariams su kompleksiniais koeficientais ir surastos ją atitinkančios sritys specialioje kompleksinio daugianario koeficientų fazinėje plokštumoje. 